\title{
Correction to: No difference in mid-term survival and clinical outcome between patient-specific and conventional instrumented total knee arthroplasty: a randomized controlled trial
}

\author{
M. G. M. Schotanus ${ }^{1}$ (D) - B. Boonen ${ }^{1} \cdot$ W. van der Weegen ${ }^{2} \cdot$ H. Hoekstra ${ }^{2} \cdot$ R. van Drumpt ${ }^{2} \cdot$ R. Borghans ${ }^{3} \cdot$ R. Vos $^{4} \cdot$ \\ L. van Rhijn ${ }^{5}$ N. P. Kort ${ }^{6}$
}

Published online: 24 May 2018

(c) European Society of Sports Traumatology, Knee Surgery, Arthroscopy (ESSKA) 2018

\section{Correction to: \\ Knee Surgery, Sports Traumatology, Arthroscopy \\ https://doi.org/10.1007/s00167-018-4968-5}

Unfortunately, one of the co-author's (W. van der Weegen) middle name has been missed in the publication of the article. The correct complete name should be W. van der Weegen.

The original article has been updated accordingly.

The original article can be found online at https://doi.org/10.1007/ s00167-018-4968-5.

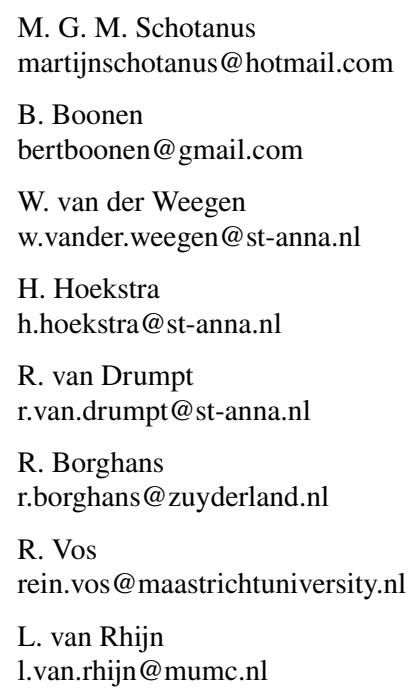

N. P. Kort

nanne@nannekort.com

1 Department of Orthopaedic Surgery and Traumatology, Zuyderland Medical Center, $\mathrm{H}$ vd Hoffplein 1, 6162 AG Sittard-Geleen, The Netherlands

2 Department of Orthopedic Surgery and Traumatology, St. Anna Hospital, Geldrop, The Netherlands

3 Department of Radiology, Zuyderland Medical Center, Sittard-Geleen, The Netherlands

4 Department of Methodology and Statistics, Maastricht University Medical Center, Maastricht, The Netherlands

5 Department of Orthopedic Surgery and Traumatology, Maastricht University Medical Center, Maastricht, The Netherlands

6 Roosteren, The Netherlands 